\title{
Design and methodology of the chronic kidney disease as a dysmetabolic determinant of disability among older people (CKD-3D) study: a multicenter cohort observational study
}

\author{
Andrea Corsonello ${ }^{1,4} \cdot$ Francesco Mattace-Raso $^{2} \cdot$ Lisanne Tap $^{2} \cdot$ Marcello Maggio $^{3} \cdot$ Luna Zerbinati $^{3}$. \\ Francesco Guarasci ${ }^{4} \odot$. Annalisa Cozza ${ }^{4}$. Sonia D'Alia ${ }^{4}$. Luca Soraci ${ }^{1,5}$. Valentina Corigliano ${ }^{1,5} \cdot$ Mirko Di Rosa $^{4}$. \\ Paolo Fabbietti ${ }^{4}$. Fabrizia Lattanzio ${ }^{6}$
}

Received: 5 September 2020 / Accepted: 5 November 2020 / Published online: 3 January 2021

(c) Springer Nature Switzerland AG 2021

\begin{abstract}
Background Chronic kidney disease (CKD) is a common condition in older people and represents a global health issue since it increases the risk of associated comorbidities and all-cause mortality. Furthermore, older people with reduced renal function might be at higher risk for developing functional limitation and disability. Moreover, the current creatinine-based measures of renal function are influenced by several factors in older population. The aims of the CKD-3D project are to perform an observational study to expand the knowledge about CKD-disability relationship and to investigate the use of novel biomarkers of kidney function.

Methods An observational, multicenter, prospective cohort study will be conducted in $75+$ old patients consecutively admitted to acute care wards of geriatric medicine at participating hospitals. The study planned to enroll 440 patients undergoing clinical and laboratory evaluations at baseline and after 12 months. Face-to-face follow-up at 6 months and telephone followup at 3 and 9 months will be carried out. Comprehensive Geriatric Assessment (CGA) and the measurement of Cystatin C, Beta-Trace Protein and Beta2-Microglobulin levels will be included.

Discussion This study will provide useful information to prevent CKD-related disability by collecting real-life data over 1-year period. The combined approach of CGA and the investigation of innovative existing biomarkers will make it possible to develop new recommendations and guidelines for a patient-centered approach. It is believed that such a study may lead to an improvement of knowledge on CKD in elderly patients and may also have implications in daily clinical practice and in decision-making process.
\end{abstract}

Keywords Chronic kidney disease $\cdot$ Disability $\cdot$ Older patients $\cdot$ Physical performance

\section{Background}

Francesco Guarasci

f.guarasci@inrca.it

Unit of Geriatric Medicine, IRCCS INRCA, Cosenza, Italy

2 Section of Geriatric Medicine, Department of Internal Medicine, Erasmus MC, University Medical Center, Rotterdam, The Netherlands

3 Geriatric Clinic Unit, Department of Medicine and Surgery, University-Hospital and University of Parma, Parma, Italy

4 Unit of Geriatric Pharmacoepidemiology and Biostatistics, IRCCS INRCA, Ancona and Cosenza, Italy

5 Department of Clinical and Experimental Medicine, University Hospital of Messina, Messina, Italy

6 Scientific Direction, IRCCS INRCA, Ancona, Italy
Chronic kidney disease (CKD) is highly prevalent affecting about $12 \%$ of the general population [1] and up to $20 \%$ of people in the age group of 60-69 years [2]; it becomes even more common in subjects over 80 years old, with estimated rates of ten times greater than those of younger adults (18-50 years old) [3]. A recent report of the Global Burden of Disease 1990-2017 showed that CKD significantly contributes to the global disease burden, and its impact on prognosis is not declining to the same extent as other highly prevalent non-communicable diseases [4].

As a consequence of the ageing of the general population, CKD is becoming a major global public health problem, imposing a substantial economic burden on health 
care systems worldwide [5]. Nowadays, CKD is considered a disease multiplier, because it increases the risk of other common associated comorbidities [6] including acute kidney injury [7], cognitive decline [8], mineral and bone disorders [9], chronic obstructive pulmonary disease (COPD) [10], anemia [11], and all-cause and cardiovascular mortality [12-14].

Early recognition of CKD through the implementation of an adequate screening and intervention strategy has shown to slow the progression of the disease and improve clinical outcomes especially among vulnerable older patients [15]. However, it may not be easy to distinguish ageing kidney from CKD, because they share many functional features, such as glomerular filtration rate (GFR) decline and impairment in tubular salt and water reabsorption [16]. Moreover, even in the absence of a clinically evident nephropathy, normal age-related changes in kidney function might be clinically relevant in the older patients [17]. In addition, it is worth considering that currently available creatinine-based measures of kidney function are plagued by some degree of inaccuracy in elderly populations due to individual anthropometric changes and to the influence of non-GFR determinants of circulating creatinine [18-21]. Indeed, several studies showed the existence of a U-shaped relationship between creatinine-based eGFR and mortality in frail and older people [22-25]. In particular, patients with high GFR values were found to be characterized by low hand grip strength and high prevalence of disability, suggesting that serum creatinine may be low due to sarcopenia rather than normal kidney function [26].

Other filtration markers which are not affected by muscle mass may better predict negative outcomes and are worth of testing [27-29]. Moreover, since CKD is often asymptomatic in older people, the assessment of kidney function is recommended especially in age group with known risk factors for CKD [30].

Older individuals with CKD might be at higher risk for developing functional limitation and disability [31]. Data from the InCHIANTI study showed that eGFR, as determined by Modification of Diet in Renal Disease (MDRD)- or CG- equations, could predict decline in activities of daily living score (ADL) over a period of 6 years in communityliving older people [32]. Another study using MDRD equation reported similar results in community-dwelling older adults over a 2-years follow-up [33].

Reduced renal function was also found to affect physical performance (e.g. Short Physical Performance BatterySPPB) [34], frailty [35], cognitive impairment [36, 37], sensory impairment [38, 39], malnutrition [40], and sarcopenia [41]. GFR as determined by Chronic Kidney Disease Epidemiology Collaboration (CKD-EPI) equation was associated with a lower SPPB total score in older hospitalized patients [32], while highest levels of Cystatin C correlated with a higher risk of self-reported functional limitation over a 1-year follow-up in community dwelling older people [42, 43]. Additionally, impaired kidney function (assessed by mGFR, eGFR or eGFRcys) was found significantly associated with self-reported frailty among participants in the MDRD study [44].

Despite the large amount of epidemiological evidence, there is a need for a comprehensive study aimed at investigating CKD among older patients and taking into account innovative biomarkers, as well as the numerous functional dimensions that CKD is able to impair.

Innovative biomarkers of kidney function, such as Cystatin C, Beta-trace Protein and B2-microglobulin, are low molecular weight proteins which are less or not affected by muscle mass and may better predict negative outcomes compared to serum creatinine among older people [45-47].

At this moment, we have no information on determinants, trajectories, and interacting factors of CKD-related disability. Hence, a prospective observational study in hospitalized patients older than 75 years, including the measure of innovative biomarkers and the systematic use of Comprehensive Geriatric Assessment (CGA) will fill this gap. Moreover, this study will not only assess the technology able to capture the numerous dimensions of health status and their complex interactions in older people, but it will also provide useful data for the development of proactive interventions in the community. It is believed that such a study will lead to an improvement of knowledge about CKD in older patients.

\section{Methods}

\section{General objectives}

The main objective of the CKD-3D project is to build an observational study to bridge knowledge gaps about the relationship between CKD and disability. Additionally, the CKD-3D study will investigate the use of innovative biomarkers of kidney function currently not used in routine assessment of CKD, allowing to compare the accuracy of different methods in predicting trajectories of disability over time.

\section{Study design}

The CKD-3D study is an observational, multicenter, prospective cohort study aimed at identifying the CKD-related disability in patients aged 75 years or older discharged from geriatric acute care wards of participating hospitals. At the baseline, the day before discharge, participants will undergo clinical and laboratory evaluations and then will be followed up at face-to-face visits at months 6 and 12 following enrollment and through intermediate telephone follow-up at 3 and 
9 months following recruitment. Figure 1 shows the flowchart of the observational clinical study.

The study design complies with the Declaration of Helsinki and Good Clinical Practice Guidelines. The study protocol was approved by ethics committees at all participating institutions. Patients are requested to sign a written informed consent before entering the study.

\section{Study population}

Patients aged 75 years and older, consecutively admitted to acute care wards of geriatric medicine at participating hospitals, are considered eligible for inclusion. No other inclusion criteria will be considered. The study design aims at minimizing self-selection bias and enrolling real-world patients without stringent inclusion/exclusion criteria. The few exclusion criteria are: age $<75$, end-stage renal disease (ESRD) or dialysis, history of solid organ or bone marrow transplantation, active malignancy within 24 months prior to screening, heart failure NYHA IV, life expectancy less than 6 months, severe cognitive impairment (i.e. MMSE < 12), complete dependency in Basic Activity of Daily Living (BADL), patients unwilling to provide consent and those who cannot be followed up, any medical or other reason (e.g. known or suspected inability of the patients to comply with the protocol procedure) in the judgement of the investigators, that the patients is unsuitable for the study. The CKD-3D study aims at finally enrolling 440 participants. The overall design of the study is reported in Fig. 1.

\section{Study endpoints}

The primary study endpoints of the CKD-3D study are:

- incident disability, defined as the loss of independency in at least 1 BADL [48];

- objectively measured functional decline, defined as the loss of at least 1 point at the assessment of physical performance by the Short Physical Performance Battery (SPPB) [49]; such threshold was formerly recommended as a substantial meaningful change in clinical studies [50].

Secondary end-points will include: CKD complications (anemia, hyperphosphatemia, acidosis, hypoalbuminemia, hyperparathyroidism, hyperkaliemia, hypertension, cardiovascular diseases (CV)); overall and CV mortality; polypharmacy and adverse drug reactions (ADRs) [51]; overall comorbidity [52]; cognitive impairment [53]; depression [54]; malnutrition/undernutrition [55]; vision and hearing impairment [56]; lower urinary tract symptoms (LUTS) [57]; decline in muscle mass assessed by bioimpedance

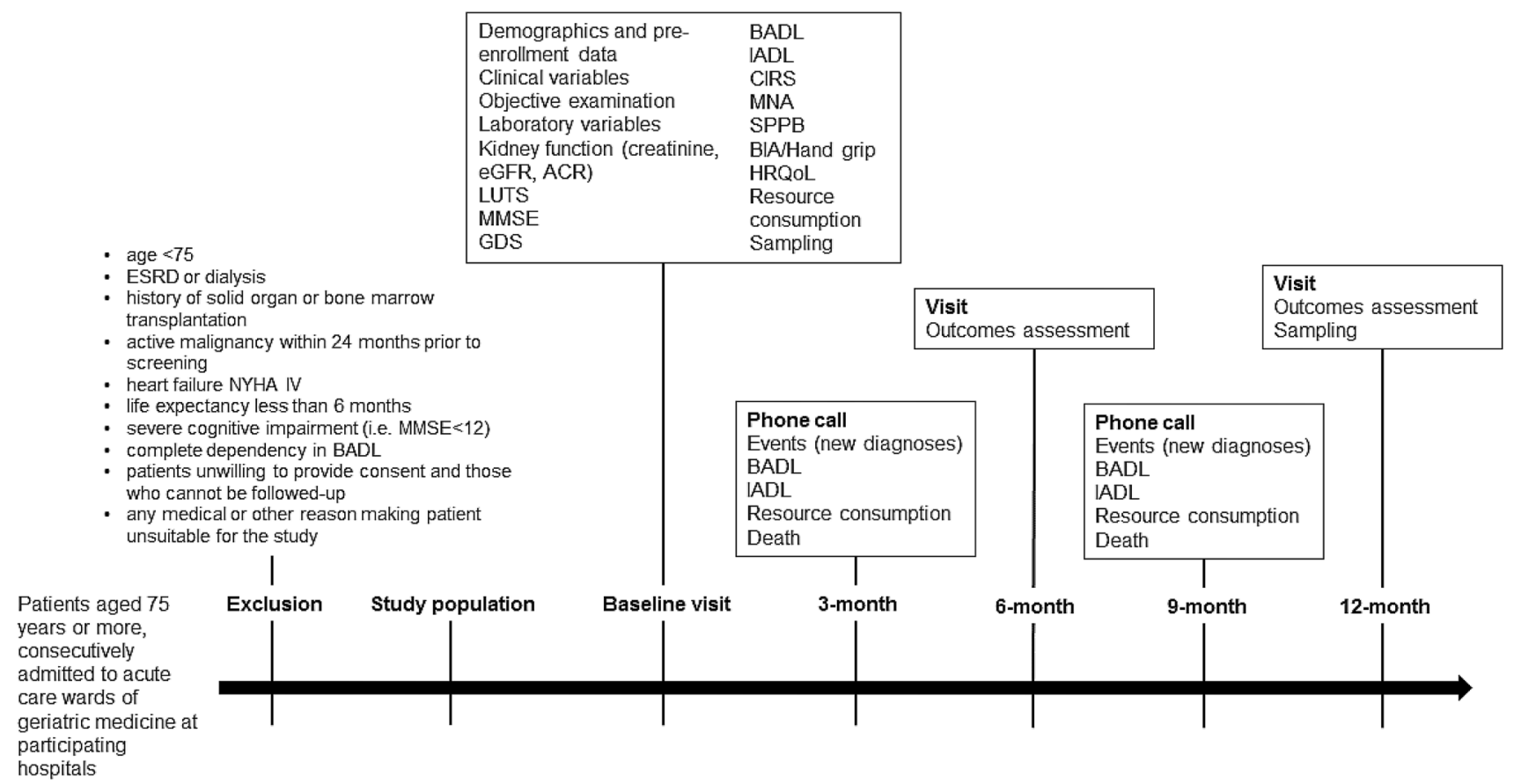

Fig. 1 CKD-3D study design. eGFR, estimated glomerular filtration rate; $A C R$, albumin-to-creatinine ratio; MMSE, mini-mental state examination; $G D S$, Geriatric Depression Scale; $B A D L$, basic activities of daily living; $I A D L$, instrumental activities of daily living;
CIRS, Cumulative Illness Rating Scale; $M N A$, mini nutritional assessment; $S P P B$, short physical performance battery; HRQoL, healthrelated quality of life 
analysis [58, 59]; hand grip strength [60]; health-related quality of life [61], healthcare resource consumption (see below); caregiver burden [62]; dependency in instrumental activities of daily living [63]. The CGA domains to be tested during the CKD-3D project are described in Table 1.

\section{Study visits}

Following enrollment, participants will be seen by the study teams at baseline (i.e. at discharge from participating wards) and at 6 and 12 months at a face-to-face meeting. Demographic data and socioeconomic status (occupation before retiring, economic status, formal and informal care) will be documented and followed up at each visit. Physical examination will be performed by medical doctors according to standardized procedure given in the visit protocol. Medical history and use of medication and adverse drug reactions classified according to the World Health Organization (WHO) definition [64] will be collected in each visit. During all face-to-face, visits a comprehensive geriatric assessment (CGA) will be performed using the instruments described in Table 1.

An ad-hoc resource use questionnaire was developed using a 3-month recall time-frame, to retrieve the following information by interviewing patient and/or caregiver: physician visits (general practitioners, specialists, or physician at the Emergency Room), use of diagnostic tests and specialist clinic procedures, use of care services (e.g. nurse home visit, physiotherapy, home help, social transport, day care center) and hospital admissions (number and duration of hospitalization, type of reimbursement).
During enrollment and 12-month follow-up visits blood and urine samples will be collected for biomarkers assessment (see "Laboratory parameters and biomarkers"). During COVID-19 outbreak, follow-up visits were stopped, and as a consequence, 67 patients were missing at FU. Nevertheless, the number of patients completing the study was 456 , which is in keeping with the sample size calculation.

\section{Telephone follow-up}

At 3 and 9 months, participants and/or caregivers will be interviewed by phone to collect information on vital and functional status and healthcare resource consumption. Changes in medical history and adverse drug reactions will also be collected.

\section{Laboratory parameters and biomarkers}

Serum creatinine measurement will be assessed by IsotopeDilution Mass Spectrometry (IDMS)-standardized method. Creatinine-based eGFR will be calculated using the Berlin Initiative Study 1 (BIS1) equation, which has been developed in a population older than 70 years [46]. ESRD will be defined as GFR $<15 \mathrm{~mL} / \mathrm{min} / 1.73 \mathrm{~m}^{2}$ or dialysis [65].

The panel of laboratory parameters to be measured at baseline, 6 and 12 months by local laboratories will also include: complete blood cells count, lipids profile, electrolytes, nutritional status, serum creatinine, urinary albumin and albumin-to-creatinine ratio and urine analysis.

Blood and urine samples will be collected at the time of discharge and at the last follow-up visit, processed and shipped frozen to the INRCA BioGer biobank, where

Table 1 Comprehensive geriatric assessment domains to be tested during the CKD-3D project

Basic (ADL) and instrumental activities of daily living (IADL)/self-reported disability [48, 49, 63]

Mini-mental state examination (MMSE)/cognitive status [53]

15-items Geriatric Depression Scale (GDS)/mood [54]

Cumulative Illness Rating Scale (CIRS)/overall comorbidity [52]

History of falls and incident falls

Vision and hearing impairment will be coded on a scale from 0 (adequate) to 4 (no vision/hearing present) [56]

Lower urinary tract symptoms (LUTS): The presence of LUTS will be ascertained by asking the patient to rate on a 5-point (0-4) Likert scale how big a problem, if any, has each of the following items been during the last 4 weeks: 1. dripping or leaking urine, 2. pain or burning in urination, 3. bleeding with urination, 4 . weak urine stream or incomplete emptying, 5. waking up to urinate, 6 . need to urinate frequently during the day [57]

Nutritional status: anthropometric parameters (calf circumference, arm circumference, body mass index $\left(\mathrm{kg} / \mathrm{m}^{2}\right)$, waist-hip ratio, waist-to-height ratio), mini nutritional assessment (MNA) [55]

Short physical performance battery (SPPB) [49]

Grip strength [60] measured using JAMAR hydraulic dynamometer

Bioelectrical impedance analysis (BIA) ${ }^{\mathrm{a}}$ [58]. Muscle mass will be calculated using the Janssen et al. equation, using the instrument Akern BIA101

Health-related quality of life will be rated by the Euro-QoL 5D [61]

${ }^{a}$ BIA will not be performed in patients with pacemaker or implantable cardioverter defibrillator 
Cystatin C (CysC) [66], Beta-Trace Protein (BTP) and Beta2-Microglobulin will be measured using standardized methods [27, 28].

\section{Sample size and statistics}

The CKD-3D project will enroll a total of 440 participants. Considering that $33 \%$ of older patients discharged from hospital experience the loss of dependency in at least $1 \mathrm{BADL}$ during a 12-month follow-up period [67], a sample of 368 patients will allow to detect a hazard ratio of 1.3 in time-toevent analyses with $80 \%$ power (alpha $=0.05$ ) for the incidence of loss of at least 1 BADL. The same sample will allow to detect a 1-point $(\mathrm{SD}=1.48)$ decline (i.e. substantial meaningful change) at SPPB measurement with $80 \%$ power in a between-group analysis [50]. Finally, even a 20\% drop out rate will not affect the statistical power of the study.

Statistical analysis will include descriptive analysis, logistic and linear regression, Kaplan-Meier and Cox regression analysis. The accuracy of different measures of kidney function in predicting outcomes will be investigated by calculating the area (AUC) under the receiver operating characteristic (ROC) curve, sensitivity, specificity, positive predictive value (PPV), negative predictive value (NPV), and $\mathrm{C}$ statistic.

Main predictors of CKD-related health-care resource consumption will be also investigated.

\section{Discussion}

The CKD-3D study is a prospective observational cohort study aimed at screening for CKD among older patients discharged from acute care wards, planned to build a prospective observational cohort database of older patients discharged from hospitals. The current paper outlines the study protocol including statistical analysis of data and risk prediction modeling related to CKD during the advanced ageing process. The strength of the protocol outlined in this paper is the real-life setting for recruitment of participants. Considering that such a high-risk population is usually affected by multiple chronic diseases, the CKD-3D study aims at minimizing selection bias by choosing broad and non-restrictive inclusion criteria: all patients with age $\geq 75$ years consecutively admitted to participating wards will be requested to participate in the study. Consequently, study results may reasonably be generalized to the whole population of older patients discharged from hospital. This is the primary strength of the CKD-3D study. The collection of real-life data in a longitudinal fashion over a 1-year period of time will allow to test the relationship between CKD and disability in the context of a real-world population, to measure outcomes relevant to older populations by the systematic use of CGA, and to compare the ability of current and innovative biomarkers in predicting trajectories of disability over time.

With these features, CKD-3D study will not only significantly impact current knowledge about CKD in older people, but it will also promote innovation in this area.

The CKD-3D project, in fact, has a high-impact translational relevance. It has been shown that CKD is associated with important changes in body function and structure able to impair physical performance and to increase the risk of disability, as well as other important geriatric outcomes. Consequently, a project based on laboratory parameters and CGA has the potential to improve the approach to CKD in older patients discharged from acute care hospitals, allowing a more patient-centered and individualized approach for screening and advanced care planning for older subjects prone to kidney function decline [68].

Findings from the CKD-3D project will significantly impact the national health system by shedding light on the complex interactions between CKD and disability, and would represent the epidemiological and methodological basis for strategic plans aimed at preventing CKD-related disability. Ultimately, the CKD-3D study will provide important information about the main predictors of costs in $\mathrm{CKD}$, which will be of paramount importance for the national health system.

In conclusion the CKD-3D project, through the use of CGA and the concurrent investigation of existing biomarkers, will be able to build new evidence in the development of recommendations and guidelines for a patient-centered approach in the screening and management of older people at risk for CKD.

Acknowledgements $C K D-3 D$ study investigators: IRCCS INRCA. Principal investigator: Andrea Corsonello. Scientific Director: Fabrizia Lattanzio. Coordinating center staff: Sabrina Garasto, Annalisa Cozza, Francesco Guarasci, Sonia D’Alia, Luca Soraci, Valentina Corigliano, Lucia Crescibene, Ramona Caloiero, Silvia Bustacchini, Silvia Bolognini, Paola D'Ascoli, Raffaella Moresi, Giuseppina Di Stefano. Technical and statistical support: Romano Firmani, Moreno Nacciariti, Mirko Di Rosa, Paolo Fabbietti. Erasmus MC, Rotterdam. Section of Geriatric Medicine, Department of Internal Medicine, Erasmus MC, University Medical Center Rotterdam, The Netherlands: Francesco Mattace-Raso, Lisanne Tap. University of Parma. Geriatric Clinic Unit, UniversityHospital of Parma and Department of Medicine and Surgery, University of Parma, Parma, Italy: Marcello Maggio, Luna Zerbinati, Giulia Ravazzoni.

Author contributions $\mathrm{AC}$ and FL conceived the study, coordinated study protocol and data collection, participated in manuscript drafting and revising. MDR and PF participated in study protocol design, data analysis, writing of the manuscript. FG taking responsibility for the publication process. FMR, LT, MM, LZ, AC, FG, SD, LS and VC participated in study protocol design, data collection, and manuscript revision and approval. All authors have read and approved the manuscript.

Funding The CKD-3D project was funded by the Grant PE-2013022357826 from Italian Ministry of Health. Funding body had no role 
in the design of the study and collection, analysis, and interpretation of data and in writing the manuscript.

Availability of data and materials The datasets generated and/or analysed during the current study will be locally available for participating researchers.

\section{Compliance with ethical standards}

Conflict of interest The authors declare no competing interests.

Ethics approval and consent to participate The study protocol was approved by ethics committees at all participating institutions, and complies with the Declaration of Helsinki and Good Clinical Practice Guidelines. All patients signed a written informed consent to be enrolled. Only baseline data are used in the present study. Ethics approvals have been obtained by Ethics Committees in participating institutions as follows: Italian National Research Center on Aging (INRCA), Italy, \#179/65/2016, University of Parma, Parma, Italy, \#33968/2017.

\section{References}

1. Hill NR, Fatoba ST, Oke JL et al (2016) Global prevalence of chronic kidney disease-a systematic review and meta-analysis. PLoS One 11:e0158765

2. Aiello F, Duenas EP, Musso CG (2017) Senescent nephropathy: the new renal syndrome. Healthcare (Basel). https://doi. org/10.3390/healthcare5040081

3. Mora-Gutierrez JM, Slon Roblero MF, Castano Bilbao I et al (2017) Chronic kidney disease in the elderly patient. Rev Esp Geriatr Gerontol. 52:152-8

4. Collaboration GBDCKD (2020) Global, regional, and national burden of chronic kidney disease, 1990-2017: a systematic analysis for the Global Burden of Disease Study 2017. Lancet 395:709-733

5. Jha V, Garcia-Garcia G, Iseki K et al (2013) Chronic kidney disease: global dimension and perspectives. Lancet 382:260-272

6. Ramer SJ, McCall NN, Robinson-Cohen C et al (2018) Health outcome priorities of older adults with advanced CKD and concordance with their nephrology providers' perceptions. J Am Soc Nephrol 29:2870

7. Hsu RK, Hsu C-Y (2016) The role of acute kidney injury in chronic kidney disease. Semin Nephrol 36:283-292

8. Drew DA, Weiner DE, Sarnak MJ (2019) Cognitive impairment in CKD: pathophysiology, management, and prevention. Am J Kidney Dis 74:782-790

9. Hou YC, Lu CL, Lu KC (2018) Mineral bone disorders in chronic kidney disease. Nephrol (Carlton) 23:88-94

10. Trudzinski FC, Alqudrah M, Omlor A et al (2019) Consequences of chronic kidney disease in chronic obstructive pulmonary disease. Respir Res 20:151

11. Cases A, Egocheaga MI, Tranche $S$ et al (2018) Anemia of chronic kidney disease: protocol of study, management and referral to Nephrology. Nefrol (Engl Ed) 38:8-12

12. Levin A (2018) Improving global kidney health: international society of nephrology initiatives and the global kidney health atlas. Ann Nutr Metab 72:28-32

13. Guo Y, Cui L, Ye P et al (2018) Change of kidney function is associated with all-cause mortality and cardiovascular diseases: results from the kailuan study. J Am Heart Assoc 7:e010596
14. Shastri S, Katz R, Rifkin DE et al (2012) Kidney function and mortality in octogenarians: cardiovascular health study all stars. J Am Geriatr Soc 60:1201-1207

15. Greer R, Boulware LE (2015) Reducing CKD risks among vulnerable populations in primary care. Adv Chronic Kidney Dis 22:74-80

16. Aucella F, Corsonello A, Leosco D et al (2019) Beyond chronic kidney disease: the diagnosis of renal disease in the Elderly as an unmet need. A position paper endorsed by Italian Society of Nephrology (SIN) and Italian Society of Geriatrics and Gerontology (SIGG). J Nephrol. 32:165-76

17. Denic A, Glassock RJ, Rule AD (2016) Structural and functional changes with the aging kidney. Adv Chronic Kidney Dis 23:19-28

18. Pedone C, Corsonello A, Incalzi RA et al (2006) Estimating renal function in older people: a comparison of three formulas. Age Ageing 35:121-126

19. Corsonello A, Pedone C, Lattanzio F et al (2011) Agreement between equations estimating glomerular filtration rate in elderly nursing home residents and in hospitalised patients: implications for drug dosing. Age Ageing 40:583-589

20. Fernandez-Prado R, Castillo-Rodriguez E, Velez-Arribas FJ et al (2016) Creatinine clearance is not equal to glomerular filtration rate and cockcroft-gault equation is not equal to CKD-EPI collaboration equation. Am J Med 129:1259-1263

21. Karger AB, Inker LA, Coresh J et al (2017) Novel filtration markers for GFR estimation. EJIFCC 28:277-288

22. Cox HJ, Bhandari S, Rigby AS et al (2008) Mortality at low and high estimated glomerular filtration rate values: a 'U'shaped curve. Nephron Clin Pract 110:c67-c72

23. Tonelli M, Klarenbach SW, Lloyd AM et al (2011) Higher estimated glomerular filtration rates may be associated with increased risk of adverse outcomes, especially with concomitant proteinuria. Kidney Int 80:1306-1314

24. Peters R, Beckett N, Poulter R et al (2013) Kidney function in the very elderly with hypertension: data from the hypertension in the very elderly (HYVET) trial. Age Ageing 42:253-258

25. Corsonello A, Roller-Wirnsberger R, Di Rosa M et al (2018) Estimated glomerular filtration rate and functional status among older people: a systematic review. Eur J Internal Med 56:39-48

26. Montesanto A, De Rango F, Berardelli M et al (2014) Glomerular filtration rate in the elderly and in the oldest old: correlation with frailty and mortality. Age (Dordr) 36:9641

27. Steubl D, Inker LA (2018) How best to estimate glomerular filtration rate? Novel filtration markers and their application. Curr Opin Nephrol Hypertens 27:398-405

28. Foster MC, Inker LA, Levey AS et al (2013) Novel filtration markers as predictors of all-cause and cardiovascular mortality in US adults. Am J Kidney Dis 62:42-51

29. George JA, Gounden V (2019) Novel glomerular filtration markers. Adv Clin Chem 88:91-119

30. Mathew TH, Corso O, Ludlow M et al (2010) Screening for chronic kidney disease in Australia: a pilot study in the community and workplace. Kidney Int Suppl 116:S9-16

31. Segura-Orti E, Gordon PL, Doyle JW et al (2018) Correlates of physical functioning and performance across the spectrum of kidney function. Clin Nurs Res 27:579-596

32. Lattanzio F, Corsonello A, Abbatecola AM et al (2012a) Relationship between renal function and physical performance in elderly hospitalized patients. Rejuvenat Res 15:545-552

33. Bowling CB, Sawyer P, Campbell RC et al (2011) Impact of chronic kidney disease on activities of daily living in community-dwelling older adults. J Gerontol Ser A Biomed Sci Med Sci 66:689-694

34. Lattanzio F, Corsonello A, Abbatecola AM et al (2012b) Relationship between renal function and physical performance in elderly hospitalized patients. Rejuvenat Res 15:545-552 
35. Walker SR, Gill K, Macdonald K et al (2013) Association of frailty and physical function in patients with non-dialysis CKD: a systematic review. BMC Nephrol 14:228

36. Drew DA, Weiner DE, Sarnak MJ. Cognitive impairment in CKD: pathophysiology, management, and prevention. Am J Kidney Dis. 2019.

37. Reckert A, Hinrichs J, Pavenstaedt $\mathrm{H}$ et al (2013) Prevalence and correlates of anxiety and depression in patients with end-stage renal disease (ESRD). Zeitschrift fur Psychosomatische Medizin und Psychotherapie 59:170-188

38. Lin C, Hsu HT, Lin YS, Weng SF (2013) Increased risk of getting sudden sensorineural hearing loss in patients with chronic kidney disease: a population-based cohort study. The Laryngoscope 123:767-773

39. Wong CW, Lamoureux EL, Cheng C-Y et al (2016) Increased burden of vision impairment and eye diseases in persons with chronic kidney disease - a population-based study. EBioMedicine 5:193-197

40. Kalender B. Malnutrition in chronic kidney disease and relationship to quality of life. In: Preedy VR, Watson RR, (eds) Handbook of disease burdens and quality of life measures. Springer, New York; 2010. p. 3159-70.

41. Ortiz A, Sanchez-Niño MD (2019) Sarcopenia in CKD: a roadmap from basic pathogenetic mechanisms to clinical trials. Clin Kidney J 12:110-112

42. Fried LF, Lee JS, Shlipak M et al (2006) Chronic kidney disease and functional limitation in older people: health, aging and body composition study. J Am Geriatr Soc 54:750-756

43. Hart A, Paudel ML, Taylor BC et al (2013) Cystatin C and frailty in older men. J Am Geriatr Soc 61:1530-1536

44. Delgado C, Grimes BA, Glidden DV et al (2015) Association of frailty based on self-reported physical function with directly measured kidney function and mortality. BMC Nephrol 16:203

45. Inker LA, Tighiouart H, Coresh J et al (2016) GFR estimation using beta-trace protein and beta2-microglobulin in CKD. Am J Kidney Dis 67:40-48

46. Schaeffner ES, Ebert N, Delanaye P, et al (2012) Two novel equations to estimate kidney function in persons aged 70 years or older. Ann Intern Med 157:471-481

47. Inker LA, Schmid CH, Tighiouart $\mathrm{H}$ et al (2012) Estimating glomerular filtration rate from serum creatinine and cystatin $\mathrm{C}$. N Engl J Med 367:20-29

48. Katz S, Ford AB, Moskowitz RW et al (1963) Studies of illness in the aged: the index of ADL: a standardized measure of biological and psychosocial function. JAMA 185:914-919

49. Guralnik JM, Fried LP, Salive ME (1996) Disability as a public health outcome in the aging population. Annu Rev Public Health 17:25-46

50. Perera S, Mody SH, Woodman RC et al (2006) Meaningful change and responsiveness in common physical performance measures in older adults. J Am Geriatr Soc 54:743-749

51. Carosella L, Pahor M, Pedone C et al (1999) Pharmacosurveillance in hospitalized patients in Italy. Study design of the 'Gruppo Italiano di Farmacovigilanza nell'Anziano' (GIFA). Pharmacol Res 40:287-295
52. Fortin M, Steenbakkers K, Hudon C et al (2011) The electronic cumulative illness rating scale: a reliable and valid tool to assess multi-morbidity in primary care. J Eval Clin Pract 17:1089-1093

53. Folstein MF, Folstein SE, McHugh PR (1975) "Mini-mental state": a practical method for grading the cognitive state of patients for the clinician. J Psychiatr Res 12:189-198

54. Lesher EL, Berryhill JS (1994) Validation of the geriatric depression scale-short form among inpatients. J Clin Psychol $50: 256-260$

55. Cereda E (2012) Mini nutritional assessment. Curr Opin Clin Nutr Metab Care 15:29-41

56. Yamada Y, Vlachova M, Richter T et al (2014) Prevalence and correlates of hearing and visual impairments in European nursing homes: results from the SHELTER study. J Am Med Direct Assoc 15:738-743

57. Rosenberg M, Staskin D, Kaplan S et al (2007) A practical guide to the evaluation and treatment of male lower urinary tract symptoms in the primary care setting. Int J Clin Pract 61:1535-1546

58. Janssen I, Heymsfield SB, Baumgartner RN et al (2000) Estimation of skeletal muscle mass by bioelectrical impedance analysis. J Appl Physiol 89:465-471

59. Binay Safer V, Geler KD (2015) Bioimpedance analysis and frailty. J Am Geriatr Soc 63:1050

60. Bohannon RW (2001) Dynamometer measurements of handgrip strength predict multiple outcomes. Percept Mot Skills 93:323-328

61. Balestroni G, Bertolotti G (2012) EuroQol-5D (EQ-5D): an instrument for measuring quality of life. Monaldi Arch Chest Dis 78:155-159

62. Yap P (2010) Validity and reliability of the Zarit Burden Interview in assessing caregiving burden. Ann Acad Med Singap 39:758-763

63. Lawton MP, Brody EM (1969) Assessment of older people: selfmaintaining and instrumental activities of daily living. Gerontol 9:179-186

64. Edwards IR, Aronson JK (2000) Adverse drug reactions: definitions, diagnosis, and management. Lancet 356:1255-1259

65. Levin A, Bilous R, Coresh J (2013) Chapter 1: Definition and classification of CKD. Kidney Int Suppl 3:19-62

66. Onopiuk A, Tokarzewicz A, Gorodkiewicz E (2015) Cystatin C: a kidney function biomarker. Adv Clin Chem 68:57-69

67. Buurman BM, Hoogerduijn JG, de Haan RJ et al (2011) Geriatric conditions in acutely hospitalized older patients: prevalence and one-year survival and functional decline. PloS One 6:e26951

68. Pilotto A, Sancarlo D, Franceschi M et al (2010) A multidimensional approach to the geriatric patient with chronic kidney disease. J Nephrol 23:S5-10

Publisher's Note Springer Nature remains neutral with regard to jurisdictional claims in published maps and institutional affiliations. 